A. Weisbecker, M. Burmester \& A. Schmidt (Hrsg.): Mensch und Computer 2015 Workshopband, Stuttgart: Oldenbourg Wissenschaftsverlag, 2015, S. 317-323.

\title{
GenImpro: Analysewerkzeug, Forschungsumgebung und generatives System für musikalische Evolution
}

\author{
Sebastian Trump \\ Hochschule für Musik Nürnberg
}

\section{Zusammenfassung}

An der Hochschule für Musik Nürnberg wird aktuell das Forschungsprojekt „Genetische Improvisation“ durchgeführt, das nach einer evolutionären Beschreibung für musikalische Entwicklungslogik sucht. In diesem Rahmen entsteht die Software-Sammlung GenImpro, die zunächst nur zur Analyse und als Forschungsumgebung für die durchgeführten Testreihen dient, später die erkannten Muster aber auch generativ nutzbar machen will, indem sie zur musikalischen Metakreation in einen genetischen Algorithmus eigespeist werden.

\section{$1 \quad$ Einleitung}

Wie entwickeln sich musikalische Ideen im Verlauf einer Improvisation? Lässt sich eine Klangfolge als genetischer Code beschreiben? Diese Fragen stehen im Zentrum des laufenden Forschungsprojekts „Genetische Improvisation“, dessen Ziel es ist, Strukturen evolutionärer Entwicklungslogik in der Musik aufzuspüren und zu beschreiben. Die Schnittstelle von Musik in Evolution wurde bisher meist aus anthropologischer Perspektive über sehr lange Zeiträume betrachtet. ${ }^{1}$ Der im Gegensatz dazu mikroskopische Blick auf Entwicklungen innerhalb eines Musikstücks ist dabei nicht nur aus musikwissenschaftlicher Sicht interessant: Für die im Bereich der musical metacreation verbreiteten evolutionären Algorithmen stellt John McCormack (2005) bisher fehlende Theorien von Musik, die evolutionäre Strukturen berücksichtigen, als Desiderat heraus. Mit dem Software-Paket GenImpro entsteht ein

1 Exemplarisch hierfür: A million years of music: the emergence of human modernity (Tomlinson 2015), Origins of Music (Wallin u. a. 2001) oder Die Anfänge der Musik (Stumpf 1911) 
integriertes System zur Analyse von Forschungsdaten und künstlerischen Anwendung als generatives System.

Das Untersuchungsobjekt bilden empirische Analysen musikalischer Improvisationen. Darin zeigen sich viel stärker als in komponierter Musik - weil unmittelbar und ohne Übersetzungsleistung zu und wieder aus einem Notentext - die Prozesse bei der Entstehung und Weiterentwicklung musikalischer Ideen. In einer Laborsituation, die nur akustische Kommunikation zwischen den Probanden erlaubt, werden deren frei - unter Vorgabe einer ungefähren Dauer - improvisierten Stücke aufgezeichnet und in GenImpro analysiert. Zentral ist dabei die Rolle des Klangs und dessen medialen Charakters, der die musikalische Interaktion in zweierlei Hinsicht formt: Zum einen in seinem direkten Wirken auf den Improvisationspartner, zum anderen in größerem Zusammenhang mit semantischer Qualität als Träger musikalischer Metainformationen. Beides zusammen bildet ein in stark abstrahierten Kategorien beschriebenes Genom einer Klangfolge. In einem späteren Schritt soll es dann möglich sein, die gewonnenen Daten als hypothetisches Modell in einen genetischen Algorithmus einzuspeisen und damit dessen Voraussagegenauigkeit zu evaluieren. So bemisst sich gleichzeitig auch die Anwendbarkeit in Algorithmen der künstlichen Kreativität und damit verbunden der künstlerische Wert des computergenerierten genetischen Klangcodes.

\section{Theoretische Grundlagen}

\subsection{Musikalische Kreativität und Improvisation}

Die Frage, wie eine musikalische Idee entsteht, ist eng verknüpft mit den Bedingungen und Prinzipien musikalischer Kreativität (Sternberg 1999). Über die ganze Musikgeschichte hinweg wurden dazu Theorien und Regelwerke als Spiegel musikästhetischer Präferenzen ihrer Zeit geschaffen, die eine formalisierte Analyse im historischen Kontext ermöglichen.

Improvisation und Komposition können aus schaffenspsychologischer Sicht entgegen der üblichen polarisierenden Gegenüberstellung als zwei Varianten eines musikalischgenerativen Prozesses betrachtet werden. Je nach Genre gibt es allerdings unterschiedliche Anteile von improvisiertem und vorgegebenem Material (Lehmann 2008). Die Ausarbeitung dieses Prozesses kann direkt realisiert (improvisiert) oder schriftlich fixiert (auf dem Notenblatt) erfolgen und iterativ evaluiert werden, wobei sowohl intern erzeugte Erwartungen als auch externe Informationen verarbeitet und zu größeren Einheiten zusammengefasst werden (Lehmann, 2005). Im Falle der Improvisation bilden Erfindung, Verarbeitung und klangliche Realisation eine phänomenologische Einheit.

Dieser komplexe Prozess weist Ähnlichkeiten zu unterschiedlichen bestehenden generativen Modellen auf: Nach Johnson-Laird (2002) generiert der Spieler zunächst passende Muster, die anschließend bewertet und selektiert werden. Hier stößt man bereits auf Indizien für eine evolutionäre Entwicklung von Klangfolgen. Daneben wird angenommen, dass der Prozess auch Ähnlichkeiten zur menschlichen Sprachproduktion aufweist (Lehmann 2008). Bereits Ende der 1980er Jahre wurden von Fred Lerdahl und Ray Jackendorff (1996) Untersuchun- 
gen durchgeführt, um musikalische Formungsprinzipien analog zur in der Linguistik entwickelten Generativen Transformationsgrammatik (Chomsky, 1969)(Chomsky 1969) zu beschreiben. Bei der Improvisation sind aber auch motorische und affektive Prozesse relevant. Trainierte Bewegungsabläufe werden automatisch abgerufen, um mehr kognitive Kapazität für Planung und ästhetische Vorstellung verfügbar zu machen (Lehmann 2008).

\subsection{Algorithmische Komposition}

Nicht erst seit der Erfindung des Computers versuchen sich Komponisten an regelbasiertem Generieren musikalischen Materials. Als eines der ersten Systeme dieser Art kann sicherlich Mozarts „Musikalisches Würfelspiel“ zum Komponieren von Walzern mit zwei Würfeln durch Rekombination vorgegebener Takte gelten (Mozart 1793).

Das erste von einem Computer komponierte Stück entstand 1955 auf einem Großrechner der Universität von Illinois: die zufallsbasierte ILLIAC-Suite für Streichquartett von Leejaren Hiller und Leonard Isaacson (Ruschkowski 1998). Später wurden auch komplexere Verfahren wie Markov-Modelle möglich, die mit einer Matrix aus Zustandsübergangsgewichtungen zwischen musikalischen Einheiten, z. B. Tonhöhen, den jeweils wahrscheinlichsten musikalischen Folgeschritt berechnen und so eine automatisierte Komposition auf Basis statistischer Analysen ermöglichen (Xenakis 1971).

In den siebziger Jahren des 20. Jahrhunderts löste der deutsche Komponist Gottfried Michael König in „Projekt 2“ das Problem der vermeintlichen Unvereinbarkeit von Zufallsoperationen und ästhetischem Prinzip durch menschliche Verifikation der computergenerierten Fragmente (Ruschkowski 1998). Diese Entwicklungen weisen uns bereits den Weg hin zum zukünftig denkbaren vollständigen Abbilden der beiden genannten Teilschritte in einem System mit künstlicher Intelligenz.

\subsection{Künstliche Intelligenz in der Musikforschung}

Mit ständig steigender Computerleistung werden zunehmend auch Modelle der Musikwahrnehmung in Programmen nachgebildet. Diese Methoden der künstlichen Intelligenz (Artifical Intelligence - AI) helfen, durch die spezifischen Herangehensweisen Forschungsprobleme zu erkennen und zu lösen: Die Konzeption erfordert eine exakte Beschreibung der vorliegenden Prozesse. Durch Simulation können neue Theorien validiert werden und Computermodelle als Inspirationsquelle für neue Hypothesen dienen (Toiviainen 2008).

Im Mittelpunkt der aktuellen Forschung stehen künstliche neuronale Netzwerke, die in stark vereinfachter Form gegenüber dem biologischen Original das Zusammenspiel virtueller Neuronen (Nodes) simulieren, die nach mathematischen Algorithmen Eingabedaten verarbeiten. In The Origins of Music (2001) beschreiben Wallin et.al. den Forschungszweig der evolutionary musicology als wichtigen Baustein für das Verständnis der Evolution des Menschen, der bisher gegenüber Erkenntnissen aus der Sprachevolution nur eine untergeordnete Rolle spielte. Aus dieser Grundidee entwickelten Peter Todd und Gregory Werner (1999) ein System, das die selektive Partnerwahl durch die Vogelweibchen bei einigen Vogelarten anhand des attraktivsten männlichen Gesangs als Verbund virtueller Komponisten und Kritiker 
nachbildet. Ungeklärt bleibt bei dieser Simulation jedoch, wie die Erwartungen der weiblichen Kritiker tatsächlich entstehen, wurden sie doch hier lediglich aus Markov-Ketten von Volksliedern abgeleitet.

Dieser Frage stellt sich Eduardo Miranda (2004) in der Beschreibung eines mimetischen Modells: Ausgehend von zufälligen Tonfolgen entsteht hier durch häufig wiederholte Imitation, die sowohl Eigenschaften des menschlichen Gehörs als auch der vokalen Klangerzeugung berücksichtigt, ein Repertoire von durch simulierte soziale Interaktion erzeugten Melodien.

AI-Systeme können neben der generativen Funktion auch noch andere Aufgaben leisten: Als Schnittstelle zwischen Mensch und Maschine verbinden Score Follower-Programme live gespielte Klänge durch probabilistische Voraussagen (Hidden-Markov-Modelle) mit dem musikalischen Verlauf einer Partitur (Toiviainen 2008).

\subsection{Genetische Algorithmen}

Von besonderem Interesse für dieses Forschungsvorhaben ist ein Ansatz, der versucht, Methoden der evolutionären Informatik als generatives Prinzip zu verwenden. Genetische Algorithmen funktionieren nach der von Darwin (1859) beschriebenen Evolutionstheorie: Aus einer Population mathematischer Objekte, die durch Mutation und Rekombination erzeugt wurde, wird durch eine fitness function die beste Variante selektiert und so zu einer neuen Generation, mit der der Ablauf erneut beginnen kann. In der Informatik dient dieses Verfahren vor allem der Annäherung an Probleme, deren optimale Lösung nicht bekannt ist und nur iterativ erreicht werden kann (Goldberg 1989; Koza 1992).

In der Musik werden Einheiten wie Akkorde, Akkordfolgen oder Melodiephrasen zu einem Genom zusammengeführt und in einem genetischen Algorithmus als Modell für künstliche Kreativität verwendet (E. R. Miranda und Biles 2007). Exemplarisch kann hierfür das Programm GenJam stehen, das durch das evolutionäre Prinzip Jazz-Solos generiert (Biles 1994).

\section{GenImpro}

Der Ansatz des Forschungsprojekts „Genetische Improvisation“ kehrt das Prinzip der evolutionären Algorithmen zunächst um und begreift diese als verborgene Mechanismen, die eine menschliche Improvisation erzeugen. Mithilfe des integrierten Software-Werkzeugs GenImpro $^{2}$ können die Aufzeichnungen analysiert und zu einem Modell der Entwicklungsprinzipien angereichert werden. In einem späteren Schritt sollen anhand des entstanden Genomalgorithmus neue Strukturen erzeugt werden können. So entstehen Schnittstellen zwischen Mensch und Computer in zweierlei Hinsicht. Erstens als zeitlich entkoppelte Abstraktion von

2 Abrufbar unter https://github.com/bastustrump/genimpro 
improvisierter Musik in einer festen Systematik, zweitens in der generativen Rückkopplung in Echtzeit.

\subsection{Analyse $^{3}$}

Die Aufbereitung der aufgezeichneten Forschungsdaten erfolgt automatisiert mit Hilfe von Python-Skripten und der ,essentia“-Bibliothek (Bogdanov u. a. 2013) in mehreren Schritten: Zunächst wird die Aufnahme in einzelne Klangereignisse (sonic events) segmentiert nach mehreren klanglichen Merkmalen beschrieben (feature extraction) Das Aufzeichnen der Merkmale hat dabei zwei unterschiedliche Ziele: Zum einen soll darüber eine Vergleichbarkeit von Klängen im technischen Sinne erreicht werden, für die eine Zuordnung von Merkmalen zu bestimmten wahrnehmbaren Klangeigenschaften keine notwenige Voraussetzung ist. Zum zweiten soll damit eine Annäherung an das Timbre eines Klangs versucht werden, das als „complex set of auditory attributes” (McAdams 1999) stark an subjektive Wahrnehmung gekoppelt ist. Anschließend werden diese nach den aus psychologischen Aspekten aus der Gestalttheorie Aspekten Nähe, Ähnlichkeit und kontinuierliche Entwicklung (Wertheimer 1925) zu Klangfolgen (sequences) gruppiert. Jede Klangfolge erhält als phänotypische Beschreibung ein Set aus zusammengefassten Parametern der enthaltenen Einzelklänge.

Wenn alle Phänotypen der Testreihe vorliegen, erfolgt darüber eine Fuzzy-C-Means Clusteranalyse, die 10 Clusterzentren im Parameterraum ermittelt und so die Genomstruktur formt. Der Abstand zu den Clusterzentren bestimmt den Genotyp der einzelnen Klangfolge und kann über eine Ähnlichkeitsmatrix Aufschluss über deren Verwandtschaftsgrad (siehe Abbildung 1) geben.

\subsection{Forschungsumgebung}

Über ein interaktives, Browser-basiertes Interface können die extrahierten genetischen $\mathrm{Zu}$ sammenhänge der Aufnahmen visualisiert werden. Die Darstellung der Klangfolgen ist entweder zeitbasiert linear (siehe Abbildung 1) oder genealogisch als phylogenetischer Baum. In einer Detailansicht zeigen sich Übergänge in einzelnen Parametern sowohl der Phänotypen als auch der Genotypen, die als Basis zur Entwicklung eines Modells der genetischen Abläufe innerhalb der Improvisation ausgewertet werden können.

3 Die genaue Methodik kann anhand von iPython notebooks unter https://github.com/bastustrump/genimpro/tree/master/notebooks nachvollzogen werden 


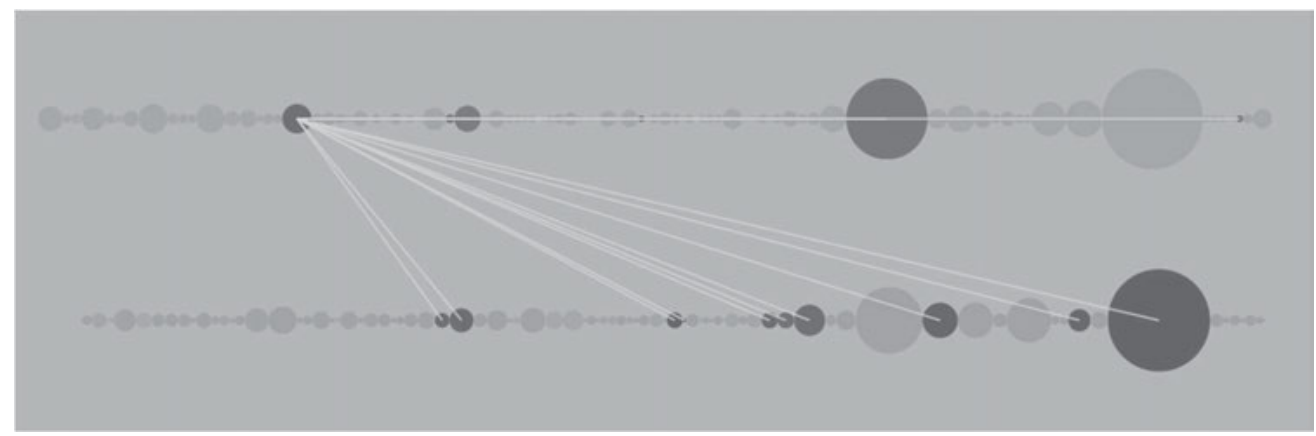

Abbildung 1: Darstellung der Klangfolgenzusammenhänge

\subsection{Metakreation}

Die Funktionen zur generativen Nutzung sind bisher noch nicht implementiert und werden erst im Laufe des Forschungsprojekts ergänzt. Geplant dafür ist eine Python-basierte ServerApplikation, die über das Open Sound Control-Protokoll (Wright 2005) angebunden werden kann und nach dem aus der Analyse ermittelten genetischen Modell neue Strukturen erzeugt. So soll es möglich sein, GenImpro an verbreitete Anwendungen wie Max/MSP, PureData, Supercollider etc. zur Klangerzeugung oder Steuerung von Live-Elektronik anzubinden.

\section{Literaturverzeichnis}

Biles, J. (1994). GenJam: A Genetic Algorithm for Generating Jazz Solos. In Proceedings of the 1994 International Computer Music Conference. Ann Arbor: University of Michigan Library.

Bogdanov, D., Wack, N., Gómez, E., Gulati, S., Herrera, P., Mayor, O., u. a. (2013). ESSENTIA: an Audio Analysis Library for Music Information Retrieval. In International Society for Music Information Retrieval Conference (ISMIR'13) (S. 493-498). Curitiba, Brazil.

Chomsky, N. (1969). Aspects of the theory of syntax. Cambridge Mass.: MIT Press.

Darwin, C. (1859). On the Origin of Species by Means of Natural Selection, or the Preservation of Favoured Races in the Struggle for Life. London: John Murray.

Goldberg, D. E. (1989). Genetic algorithms in search, optimization, and machine learning. Reading, Mass.: Addison-Wesley Pub. Co.

Johnson-Laird, P. N. (2002). How Jazz Musicians Improvise. Music Perception: An Interdisciplinary Journal, 19(3), 415-442.

Koza, J. R. (1992). Genetic programming: on the programming of computers by means of natural selection. Cambridge, Mass.: MIT Press.

Lehmann, A. C. (2008). Komposition und Improvisation. In H. Bruhn, A. C. Lehmann, \& R. Kopiez (Hrsg.), Musikpsychologie. Das neue Handbuch. Reinbek bei Hamburg: Rowohlt. 
Lerdahl, F., \& Jackendorff, R. (1996). A generative theory of tonal music. Cambridge, Mass.: MIT Press.

McAdams, S. (1999). Perspectives on the contribution of timbre to musical structure. Computer Music Journal, 23(3), 85-102. Zugegriffen 27. Februar 2015

McCormack, J. (2005). Open Problems in Evolutionary Music and Art", in F. Rothlauf et al. (eds), Lecture Notes in Computer Science, Vol 3449, Applications of evolutionary computing: EvoWorkshops 2005, EvoBIO, EvoCOMNET, EvoHOT, EvoIASP, EvoMUSART, and EvoSTOC, Lausanne, Switzerland, March 30-April 1, 2005: proceedings.

Miranda, E. (2004). At the Crossroads of Evolutionary Computation and Music: SelfProgramming Synthesizers, Swarm Orchestras and the Origins of Melody. Evolutionary Computation, 12(2), 137-158.

Miranda, E. R., \& Biles, J. (Hrsg.). (2007). Evolutionary computer music. London: Springer.

Mozart, W. A. (1793). Musikalisches Würfelspiel. Eine Anleitung „Walzer Oder Schleifer Mit Zwei Würfeln Zu Componieren Ohne Musikalisch Zu Seyn, Noch Von Der Composition Etwas Zu Verstehen “. (K. H. Taubert, Hrsg.). Mainz: Schott.

Ruschkowski, A. (1998). Elektronische Klänge und musikalische Entdeckungen. Stuttgart: Reclam.

Sternberg, R. J. (Hrsg.). (1999). Handbook of creativity. Cambridge, U.K. ; New York: Cambridge University Press.

Stumpf, C. (1911). Die Anfänge der Musik. Leipzig: J.A. Barth.

Todd, P. M., \& Werner, G. M. (1999). Musical Networks. In N. Griffith \& P. M. Todd (Hrsg.), . Cambridge, Mass.: MIT Press.

Toiviainen, P. (2008). Musikalische Wahrnehmung und Kognition im Computermodell. In H. Bruhn, A. C. Lehmann, \& R. Kopiez (Hrsg.), Musikpsychologie. Das neue Handbuch. Reinbek bei Hamburg: Rowohlt.

Tomlinson, G. (2015). A million years of music: the emergence of human modernity (First edition.). New York: Zone Books.

Wallin, N. L., Merker, B., \& Brown, S. (2001). The Origins of Music. Cambridge, Mass.: MIT Press.

Wertheimer, M. (1925). Drei Abhandlungen zur Gestalttheorie. Erlangen: Philosphische Akademie.

Wright, M. (2005). Open Sound Control: an enabling technology for musical networking. Organised Sound, 10(03), 193-200.

Xenakis, I. (1971). Formalized music. Indiana University Press.

\section{Kontaktinformationen}

Sebastian Trump, Hochschule für Musik Nürnberg, Veilhofstraße 34-40, 90489 Nürnberg, sebastian.trump@hfm-nuernberg.de, Tel. +49 (0)911/231-14972 
